\title{
Comparison between Bi-2223 Tape and RE-123 Coated Conductor from the View Point of Current Transport Properties Influencing Thermal Stability
}

\author{
Takanobu Kiss $^{1)} *$, Masayoshi Inoue ${ }^{1)}$, Kohei Higashikawa ${ }^{1)}$, Takumi Suzuki ${ }^{1)}$, Lin Lyu ${ }^{1)}$, Ken

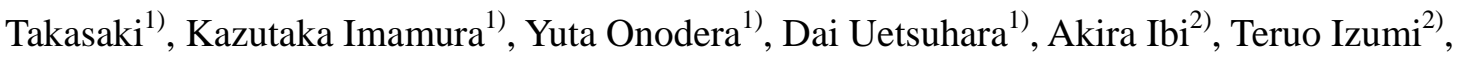 \\ Hitoshi Kitaguchi ${ }^{3)}$ \\ ${ }^{1)}$ Department of Electrical Engineering, Kyushu University, 744 Motooka, Fukuoka 819-0395, Japan \\ ${ }^{2)}$ ISTEC-SRL, 3-2-1 Sakado, Takatsu-ku, Kawasaki-Shi, Kanagawa, 213-0012 Japan \\ ${ }^{3)}$ National Institute for Materials Science, 1-2-1 Sengen, Tsukuba, Ibaraki 305-0047, Japan
}

\begin{abstract}
We have investigated flux flow dissipation in typical two kinds of HTS tapes, i.e., a Bi-2223 multi-filamentary tape and a RE-123 coated conductor (CC) from the view point of heat load under over current conditions. Based on systematic measurements on current-voltage characteristics, the nonlinear flux flow dissipation has been described analytically by taking into account current sharing in metallic sheath or stabilization layer. Flux flow dissipation in the RE-123 CC shows much steeper temperature dependence than that of the Bi-2223 tape. As a result, attainable cooling power becomes smaller in the RE-123 $\mathrm{CC}$ in comparison with that of $\mathrm{Bi}-2223$ tape even if the same cooling condition. In other word, acceptable temperature rise in the RE-123 CC is small at over current condition, whereas moderate temperature dependence in the Bi-2223 tape allows stable operation even if the bias current exceeds the critical current. Influence of spatial inhomogeneity in the both HTS tapes has also been investigated. Longitudinal variation of local critical current, $I_{\mathrm{c}}$, and its statistical behavior have been characterized by use of reel-to-reel scanning Hall probe microscopy. It has been found that the flux flow dissipation is possibly localized more than one order higher than that of the average value due to discrete local $I_{\mathrm{C}}$ drops.
\end{abstract}

Keywords: flux flow dissipation, critical current, spatial inhomogeneity, statistical distribution, Bi-2223 multi-filamentary tape, RE-123 coated conductor

Corresponding author*: kiss@sc.kyushu-u.ac.jp

\section{Introduction}

Acceptable dissipation level becomes large in a high $T_{\mathrm{c}}$ superconductor (HTS) because of its large heat capacity at an elevated operation temperature. Rounded current-voltage, $I-V$, characteristics also make the boundary between zero resistive state and dissipative state broad. As a result, heat balance between cooling and dissipation becomes more essential rather than a simple electric field criterion to determine thermal stability in the HTS. Namely, not only critical current, $I_{\mathrm{c}}$, but also $I-V$ characteristics at dissipative state becomes relevant to understand optimal cooling conditions and/or the behavior of thermal instability and switching characteristics of superconducting fault current limiters under over load conditions. However, such dissipation in a HTS tape shows non-linear dependence as a function of bias current, magnetic field and temperature in a complicated manner. Spatial inhomogeneity is also an important factor to determine thermal stability, however, the influence of local $I_{\mathrm{c}}$ variation is not yet fully understood in the HTS tapes because standard characterization techniques in a macroscopic scale cannot detect such local variation. In this study, we

\section{Cryogenics and Superconductivity Society of J apan (CSSJ )}


compared flux flow dissipation in commercially available typical two kinds of HTS tapes, i.e., Bi-2223 multi-filamentary tape and RE-123 coated conductor (CC) from the view point of heat load under over current conditions.

2. $I$ - $V$ characteristics in HTS tapes over multi-decades of voltage range

2.1. Percolation transition model in HTS materials

To describe the nonlinear $I-V$ characteristics in HTS materials, power law $I-V$ relationship is widely used. Actually, that is a good approximation in a limited voltage range, however, the power index depends on voltage range. Namely, the power law model doesn't hold in multi-decades of voltage range generally. To describe the $I-V$ characteristics in such a wide voltage range, we utilized percolation transition model in which flux penetration due to Lorentz force can be described as a kind of percolation phenomena in a disordered medium because of randomly distributed pinning centers in the HTS materials [1, 2]. As schematically shown in Fig. 1, low $J_{\mathrm{c}}$ network forms a percolation cluster if the bias current reaches threshold value, $J_{\mathrm{cm}}$. When we further increase the bias current, induced flux flow voltage increases gradually in proportion to the area size of the percolation cluster. Such two dimensional network can be converted to an equivalent series domain network in which each domain size is in proportion to the probability of critical current density, $J_{\text {ci }}$, in the corresponding domain. Therefore the global $E$ - $J$ characteristics can be obtained by integrating the whole series network by taking into account the probability density function, $P$, of the local critical current density. Here, we adopted Weibull function to describe $P\left(J_{\mathrm{ci}}\right)$ where the risk probability increases in proportion to the power of $\left(J-J_{\mathrm{cm}}\right) / J_{0}$, here $J_{0}$ is scale parameter. Since each domain has a single value of critical current density $J_{\mathrm{c}}$, we can describe elementary $E-J$ characteristics in each domain, $E_{\mathrm{e}}\left(J, J_{\mathrm{ci}}\right)$, by a simple flux flow model. As a result, the global $E-J$ characteristics can be expressed analytically as follows.

$$
\begin{aligned}
& E(J) \equiv \frac{V}{L}=\frac{1}{L} \sum_{i=1}^{n} L_{\mathrm{i}} E_{\mathrm{e}}\left(J, J_{\mathrm{ci}}\right)=\int_{J_{\mathrm{cm}}}^{\infty} P\left(J_{\mathrm{C}}\right) E_{\mathrm{e}}\left(J, J_{\mathrm{C}}\right) d J_{\mathrm{C}}, \\
& E_{\mathrm{e}}\left(J, J_{\mathrm{c}}\right)=\left\{\begin{array}{cc}
\rho_{\mathrm{FF}}\left(J-J_{\mathrm{C}}\right) & \text { for } J>J_{\mathrm{C}}, \\
0 & \text { for } J \leq J_{\mathrm{c}},
\end{array}\right. \\
& P\left(J_{\mathrm{c}}\right)=\frac{m}{J_{0}}\left(\frac{J_{\mathrm{C}}-J_{\mathrm{cm}}}{J_{0}}\right)^{m-1} \exp \left[-\left(\frac{J_{\mathrm{C}}-J_{\mathrm{cm}}}{J_{0}}\right)^{m}\right]
\end{aligned}
$$

where $\rho_{\mathrm{FF}}$ is flux flow resistivity and $m$ is power index. In a practical operation condition, the bias current density, $J$, is close to $J_{\mathrm{cm}}$, therefore, we can extend the exponential function in eq. (3), then can obtain approximation for the $E-J$ characteristics as follows.

$$
\begin{array}{rlrl}
E(J, B, T) & =\frac{\rho_{\mathrm{FF}}}{m+1} J\left(\frac{J}{J_{0}(B, T)}\right)^{m}\left(1-\frac{J_{\mathrm{cm}}(B, T)}{J}\right)^{m+1} & & \text { for } T \leq T_{\mathrm{GL}}, J>J_{\mathrm{cm}}, \\
& =0 & \text { for } T \leq T_{\mathrm{GL}}, J \leq J_{\mathrm{cm}},
\end{array}
$$




$$
E(J, B, T)=\frac{\rho_{\mathrm{FF}}}{m+1}\left|J_{\mathrm{cm}}(B, T)\right|\left(\frac{\left|J_{\mathrm{cm}}(B, T)\right|}{J_{0}(B, T)}\right)^{m}\left\{\left(1+\frac{J}{\left|J_{\mathrm{cm}}(B, T)\right|}\right)^{m+1}-1\right\} \text { for } T>T_{\mathrm{GL}} \text {, }
$$

where $T_{\mathrm{GL}}$ is the vortex-glass-liquid transition temperature. Coupling with scaling behavior of flux pinning to describe $J_{\mathrm{cm}}(B, T)$ and $J_{0}(B, T)$, we can describe the $E-J$ characteristics as a function of temperature, $T$, and magnetic field, $B$, in RE-123 thin film [1, 2], RE-123 CCs [3, 5] and $\mathrm{Bi}-2223$ tape $[3,4]$.

\subsection{Current sharing in a highly dissipative state}

In a highly dissipative state, we should take into account current sharing in metallic sheath or stabilization layer. Here, we adopt a simple parallel circuit model consists of a superconducting region and a silver region as a metallic sheath or stabilizer. Nonlinear resistance of the superconducting region, $R_{\mathrm{SC}}$, in a length, $L$, can be obtained as follows by using above mentioned eq. (4).

$$
R_{S C}=\frac{E\left(\frac{\beta I_{t}}{\hat{\alpha} A_{t}}, B, T\right)}{\beta I_{t}} L
$$

where $A_{\mathrm{t}}$ is total cross-section area of the tape, $\hat{\alpha}$ is the ratio of superconducting area in the cross-section, $I_{\mathrm{t}}$ is total current and $\beta$ is the current ratio flowing superconducting region. The resistance in the normal region, $R_{\mathrm{n}}$, is given by

$$
R_{n}=\frac{\rho_{A g}(T)}{(1-\hat{\alpha}) A_{t}} L,
$$

where $\rho_{\mathrm{Ag}}$ is temperature dependent resistivity of silver. In the case of CC, we only take into account superconducting layer and Ag stabilization layer assuming that the current through buffer template and Hastelloy substrate is negligible.

$\beta$ can be obtained numerically by the following equation as a function of $I_{\mathrm{t}}, B$ and $T$.

$$
E\left(\frac{\beta I_{t}}{\hat{\alpha} A_{t}}, B, T\right)=\rho_{A g}(T) \frac{(1-\beta) I_{t}}{(1-\hat{\alpha}) A_{t}} .
$$

Then, finally total electric field in the HTS tape can be obtained by

$$
E=\frac{1}{\frac{1}{R_{S C}}+\frac{1}{R_{n}}} \frac{I_{t}}{L}=R_{t} \frac{I_{t}}{L} .
$$

2.3. Comparison between measurements and the model: Bi-2223 tape and RE-123 CC

E-I characteristics of the both Bi-2223 tape and RE-123 CC were measured by the transport method, then compared with the model described in the previous section. Specification of the samples were summarized in Table 1.

$E-I$ characteristics in a commercially available recent 200 -A-class DI-BSCCO ${ }^{\mathrm{TM}}$ Type $\mathrm{H}$ were shown in Fig. 2, where solid curves are analytical expression whereas dots are 
measurements. Experimental data was obtained from standard four-probe method with a voltage tap distance of $1 \mathrm{~cm}$. Broken lines in the figures show equi Joule heating power line in the measurements. As can be seen in the figure, reasonable agreement between the analytical expression and measurements was obtained over wide range of electric field at various conditions of magnetic field and temperature. In the similar way, we also obtained results for a Gd-123 CC tape as shown in Fig. 3. For the measurement, we formed a 100 - $\mu \mathrm{m}$-wide and 500 - $\mu \mathrm{m}$-long microbridge by a typical photo lithography and wet etching process in order to suppress Joule heating during the measurements. Then the current was calibrated for a 4-mm-wide full width tape.

One of the important advantage of analytical approach is to be able to describe the properties at excess current conditions with highly dissipative flux flow state [6]. This is relevant especially in the $\mathrm{Bi}-2223$ tape because we cannot form a microbridge for the measurements.

\section{Thermal runaway and nonlinear flux flow dissipation}

In Fig. 4, we compared flux flow dissipation, $P_{\mathrm{F}}$, as a function of temperature for a unit tape length at a constant bias current in both Bi-2223 tape and Gd-123 CC by use of analytical formula described in the previous section. It is clearly shown that the Gd-123 CC has much steeper temperature dependence than that of the Bi-2223 tape.

In our previous study, we pointed out that thermal runaway can be described as a kind of catastrophic transition observed in a nonlinear system [6]. Temperature rise due to the over current can be expressed by the heat balance equation [7].

$$
C \frac{d T}{d t}=\frac{R_{t}}{A_{t} L} I_{t}^{2}-\frac{h a\left(T-T_{0}\right)}{A_{t}},
$$

where $C$ is heat capacity, $h$ is heat transfer coefficient for convective cooling, $a$ is perimeter of the superconducting tape exposed to the coolant, $T_{0}$ is bias temperature, and $t$ is time. For a non-uniform tape, spatial variation of the temperature and the thermal conduction along and/or across the tape should also be considered for a precise analysis, however, we ignored the thermal conduction for simplicity in the present analysis. This assumption maximizes the temperature and possibly cause slight over estimation of the critical condition discussed below but it is still a good approximation of a real situation since the thermal conductivity of the HTS tapes is usually low $[8,9]$.

Thermal runaway occurs when the Joule heating power curve loses the cross point with the cooling power line due to its nonlinearity, namely

$$
\frac{R_{t}}{L} I_{t}^{2}>h a\left(T-T_{0}\right)
$$

Therefore, stable operation condition depends strongly on the nonlinearity of the flux flow dissipation not only the cooling condition. In Figs. 5 and 6, we compared bias current dependences more precisely at $B=0.5 \mathrm{~T}$ around $T=65 \mathrm{~K}$, and $B=3.0 \mathrm{~T}$ around $T=30 \mathrm{~K}$, respectively. The convective cooling power was estimated based on our previous study [6] assuming a cooling by Helium gas flow as an example, where $h=1.25 \times 10^{3} \mathrm{~W} / \mathrm{m}^{3}$, and $a=4$ $\mathrm{mm}$. 
These results show that acceptable temperature rise during the over current operation in the Gd-123 CC tape is much smaller than that of the Bi-2223 tape, therefore attainable cooling power at the critical point is also smaller in comparison with Bi-2223 tape even if in the same cooling condition. Acceptable temperature rise and cooling power in the Gd-123 CC tape is estimated to be $1 \mathrm{~K}$ and $0.5 \mathrm{~W} / \mathrm{m}$ at $0.5 \mathrm{~T}$ and $65 \mathrm{~K}$. As a result, thermal quench current is only $8 \%$ larger than the value of $I_{\mathrm{c}}$ at the operation temperature. On the other hand, Bi-2223 tape allows $3.2 \mathrm{~K}$ of temperature rise and the attainable cooling power can be $1.6 \mathrm{~W} / \mathrm{m}$ because of its moderate temperature dependence. Quench current, therefore, can reach 2.85 times of $I_{\mathrm{c}}$ even though the same cooling condition. Similar tendency can be seen at lower temperature around $30 \mathrm{~K}$ at $3 \mathrm{~T}$. Quench current of the Gd-123 CC tape is estimated to be $5 \%$ larger than the $I_{\mathrm{c}}$ whereas $\mathrm{Bi}-2223$ tape allows excess current up to $46 \%$ larger value of $I_{\mathrm{c}}$ for a stable operation. Such thermal stability should be a good advantage of Bi-2223 tape.

\section{Influence of spatial inhomogeneity of critical currents}

In order to investigate local $I_{\mathrm{C}}$ variation in the HTS tapes with high spatial resolution, we carried out reel-to-reel scanning Hall-probe microscopy (RTR-SHPM). By traveling the tape in reel-to-reel manner, we magnetized the tape in a liquid nitrogen bath continuously, then measured remanent field distribution on the tape surface by using a high-speed scanning micro-Hall probe. Magnetization current can be obtained by solving inversion problem of Biot-Savart law. Integrating the magnetization current along the width, we can estimate $I_{\mathrm{c}}$ value as a function of longitudinal coordinate, $x$ [10]. By this method, we can detect $I_{\mathrm{c}}$ variation with a spatial resolution of $850 \mu \mathrm{m}$ in $x$ that is several order smaller than that of typical transport reel-to-reel measurement [11-13]. Thanks to such high resolution measurement, we have succeeded in detecting discrete local $I_{\mathrm{c}}$ drops in a 6-m-long Bi-2223 tape as shown in Fig. 7 (a) for the first time. The sample is the same lot studied in-field flux flow dissipation discussed in the preceding section. Electric field criterion determined from the induction voltage during the measurement was $2 \times 10^{-8} \mathrm{~A} / \mathrm{m}$ that is four orders smaller than that of typical four-probe method, therefore, measured $I_{\mathrm{c}}$ was about $30 \%$ smaller than the value obtained from transport measurement.

We also calculated global $I-V$ characteristics based on the $I_{c}(x)$ obtained from the RTR-SHPM with assumptions that 1) $I_{\mathrm{c}}$ variation observed by the RTR-SHPM comes from that of effective cross-section area of the superconductivity region due to macroscopic defects such as partial distortion of filaments and current blocking obstacles in the tape, 2) while flux pinning behavior in nano-scale is essentially the same in the whole length. Because the spatial resolution, $\Delta x=850 \mu \mathrm{m}$, of the RTR-SHPM is several orders longer than the spacing of vortices, scattering of the flux pinning strength should be averaged, therefore, it is reasonable assumption that the observed $I_{\mathrm{C}}$ by the RTR-SHPM is proportional to the average cross-section area of the superconducting region at that coordinate. This assumption has been supported by correlated study between site-specified microstructural analysis and in-field current transport measurements. Detailed analysis on this point will be published separately.

We can collect the information on spatial variation of the current blocking obstacles as a function of $x$ by introducing a parameter $\alpha(x) \equiv I_{\mathrm{c}}(x) / I_{\mathrm{c}, \max }$, where $I_{\mathrm{c}, \max }$ is the maximum value of the $I_{\mathrm{C}}(x)$. Whereas the local electric field at the coordinate $x$ can be described by the eq. (4) by taking into account $\alpha(x)$ as follows

$$
E\left(\frac{I_{t}}{\hat{\alpha} \alpha(x) A_{t}}, B, T\right) \text {. }
$$


From the discrete experimental data $I_{\mathrm{c}}\left(x_{\mathrm{j}}\right)$, we obtain $\alpha\left(x_{\mathrm{j}}\right)$ where $x_{\mathrm{j}}=j \Delta x, j=1,2, \ldots, \mathrm{N}$, and $\mathrm{N}=L / \Delta x$, then we can calculate global $E-I$ characteristics in the whole length $L$ by using following equations.

$$
\begin{gathered}
R_{S C, j}=\frac{E\left(\frac{\beta_{j} I_{t}}{\hat{\alpha} \alpha\left(x_{j}\right) A_{t}}, B, T\right)}{\beta_{j} I_{t}} \Delta x, \\
E\left(\frac{\beta_{j} I_{t}}{\hat{\alpha} \alpha\left(x_{j}\right) A_{t}}, B, T\right)=\rho_{A g}(T) \frac{\left(1-\beta_{j}\right) I_{t}}{(1-\hat{\alpha}) A_{t}}, \\
E=\sum_{j=1}^{N} \frac{1}{\frac{1}{R_{S C, j}}+\frac{1}{R_{n}}} \frac{I_{t}}{L} .
\end{gathered}
$$

As far as the induced electric field is low enough, we can ignore the current sharing to the normal layer, therefore, $\beta_{\mathrm{j}}$ should be 1 in the all section.

Analytical result on the local electric field was shown in Fig. 7 (b) at the condition where global electric field becomes $1 \mu \mathrm{V} / \mathrm{cm}$. Global $I_{\mathrm{c}}$ obtained from eq. (14) shows good agreement with that of transport measurement, $190 \mathrm{~A}$. The value of local flux-flow dissipation, q, was also shown in Fig. 7 (b) in the axis right-hand side. It can be seen that the dissipation is possibly localized at several discrete positions more than one order higher than that the average value.

Similar analysis on the spatial homogeneity has been carried out also on a 10-m-long commercial Gd-123 CC as shown in Fig. 8. Electric field criterion for the RTR-SHPM was $3 \times 10^{-8} \mathrm{~A} / \mathrm{m}$. Discrete localized electric field with a level of several tens of $\mu \mathrm{V} / \mathrm{cm}$ has been confirmed again even though the average electric field was as low as $1 \mu \mathrm{V} / \mathrm{cm}$.

Statistical distribution of $\alpha$ was shown in Fig. 9 for both Bi-2223 tape and Gd-123 CC. The probability density function shows asymmetric distribution and can be well described by the Weibull function.

$$
P(\alpha)=\left(\frac{m}{\alpha 0}\right)\left(\frac{\alpha-\alpha \min }{\alpha 0}\right)^{m-1} \exp \left[-\left(\frac{\alpha-\alpha \text { min }}{\alpha 0}\right)^{m}\right]
$$

In the case of Bi-2223 tape, we observed combination of two different distributions, $0.003 P_{1}+0.997 P_{2}$, that suggest two different origins for the current blocking obstacles such as filament sausaging and crack formation. Whereas in the Gd-123 CC, single distribution can describe the experimental result. Parameters of the distribution were listed in Table 2. It can be seen that the distribution of $\alpha$ is broader in the Bi-2223 tape than the Gd-123 CC. However, as can be seen in Figs. 7(b) and 8(b), the localization is more prominent in the Gd-123 CC than the Bi-2223 tape. This is originated from sharp $I-V$ transition in the Gd-123 CC.

5. Conclusion

Relationship between nonlinear flux flow dissipation and thermal runaway in both Bi-2223 
tape and RE-123 CC have been discussed. Nonlinear flux flow resistivity has been analyzed based on the percolation transition model proposed by the authors. It has been shown that RE-123 CC tape has much steeper temperature dependence of flux flow dissipation compared to that of $\mathrm{Bi}-2223$ tape. This indicates that acceptable temperature rise is small in the RE-123 CC tape before the thermal runaway, as a result, attainable cooling power is also small in comparison with Bi-2223 tape even if we use the same cooling condition. High resolution RTR-SHPM allows us to detect discrete local $I_{\mathrm{C}}$ drop of 20-25 \% in several positions. Based on statistical analysis of such $I_{\mathrm{c}}$ variation, we have analyzed relationship between global $I-V$ characteristics and the local one. This leads a conclusion that the flux flow dissipation is possibly localized more than one order higher than that of the average value. Such localized dissipation can reach several $\mathrm{mW} / \mathrm{cm}$ or higher at $77 \mathrm{~K}$ self-field condition though the apparent average electric field among the whole length is still as low as $1 \mu \mathrm{V} / \mathrm{cm}$. Namely, it is hardly possible to detect these localized dissipations using the standard four-probe measurement in a macroscopic scale. Such localization is more prominent in Gd-123 CC tape than $\mathrm{Bi}-2223$ tape because of its sharp $I-V$ transition, whereas the spatial uniformity of the superconducting cross-section area is better in the Gd-123 CC.

Acknowledgements: This work is supported by the AMED and the METI as a project of "Development of HTS Coiling Technology", “JST: S-Innovation” and "JSPS: KAKENHI (24360122, 26630113).”

\section{References}

[1] K. Yamafuji and T. Kiss, Physica C 258 (1996) 197-212.

[2] T. Kiss, T. Matsushita and F. Irie, SuST 12 (1999) 1079-1082.

[3] T. Kiss, M. Inoue et al, Physica C 392-396 (2003) 1053-1062.

[4] T. Kiss et al., Advances in Cryogenic Engineering: Proc. of ICMC 48 (2002) 1091-1101.

[5] T. Kiss et al., IEEE Trans. Appl. Supercond. 13 (2003) 2607-2610.

[6] T. Kiss et al., IEEE Trans. Appl. Supercond. 9 (1999) 1073-1076.

[7] R. H. Bellis and Y. Iwasa, Cryogenics 34 (1994) 129-144.

[8] P. Tixador, T. Nguyen-Nhat, H. G. Okada-Vieira, and R. Ponceau, IEEE Trans. Appl. Supercond. 21 (2011) 1194-1197.

[9] M. Iwakuma, Y. Katanosaka, S. Sato et al., IEEE Trans. Appl. Supercond. 23 (2013) 5500104.

[10] K. Higashikawa et al., IEEE Trans. Appl. Supercond. 23 (2013) 6602704.

[11] T. Nakashima et al., Cryogenics 52 (2012) 713-718.

[12] M. Igarashi et al., Physics Procedia 36 (2012) 1412-1416.

[13] J. J. Gannon, Jr., A. P. Malozemoff, R. C. Diehl, P. Antaya, and A. Mori, IEEE Trans. Appl. Supercond. 23 (2013) 8002005. 
Table 1. Specification of the sample

\begin{tabular}{lcc} 
Sample & Bi-2223 tape & Gd-123 CC \\
\hline $\begin{array}{l}I_{\mathrm{c}} \text { at } 77 \mathrm{~K}, \text { s.f. }[\mathrm{A}] \\
\left.\text { (defined at } 10^{-6} \mathrm{~V} / \mathrm{cm}\right)\end{array}$ & 190 & 240 \\
\hline Width $[\mathrm{mm}]$ & 3.8 & 4.0 \\
\hline$A_{\mathrm{t}}\left[\mathrm{mm}^{2}\right]$ & 0.912 & $* 0.208$ \\
\hline$\hat{\alpha}$ & $2.86 \times 10^{-1}$ & $* 3.85 \times 10^{-2}$ \\
\hline
\end{tabular}

* We took into account superconducting layer and Ag stabilization layer only in the case of CC assuming that the current through buffer template and Hastelloy substrate is negligible.

Table 2. Parameters of statistical distribution of $\alpha$

\begin{tabular}{llll} 
Sample & \multicolumn{2}{c}{ Bi-2223 tape } & Gd-123 CC \\
\hline Function & $0.003 P_{1}$ & $0.997 P_{2}$ & $P$ \\
\hline$m$ & 8.0 & 10.0 & 13.0 \\
\hline$\alpha_{\min }$ & 0.506 & 0.608 & 0.625 \\
\hline$\alpha_{0}$ & 0.203 & 0.304 & 0.304 \\
\hline
\end{tabular}



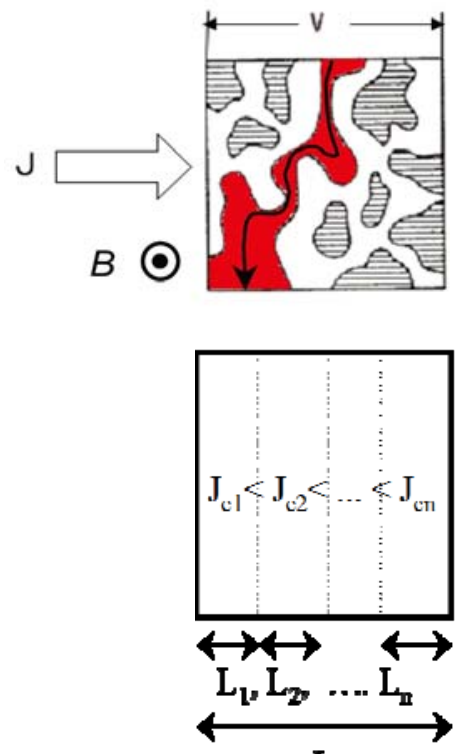

L

Fig. 1 Percolation transition model 

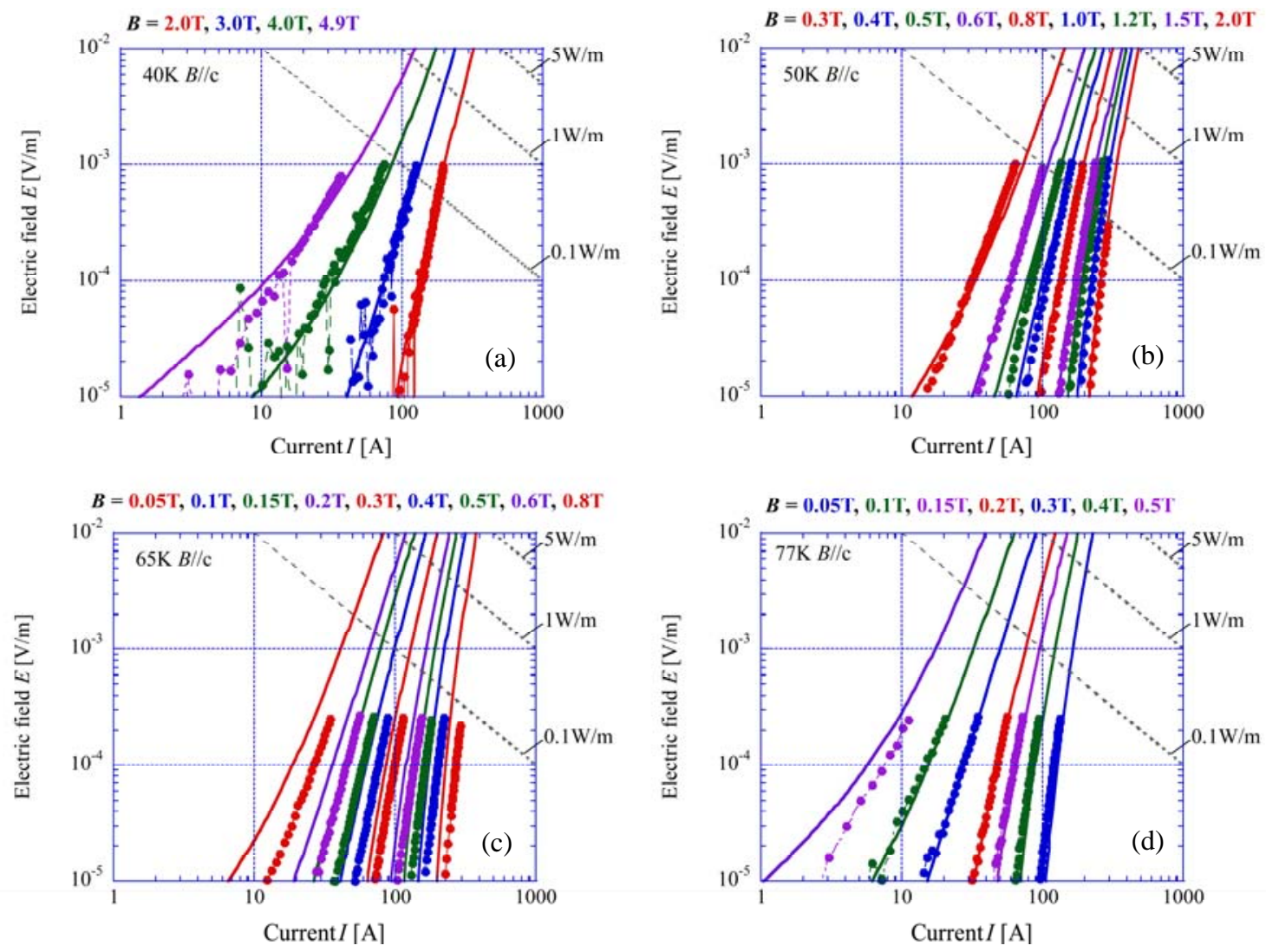

Fig. 2 E-I characteristics in a commercially available 3.8-mm-wide Bi-2223 tape. Solid curves are analytical expression by percolation transition model whereas dots are measurements. 

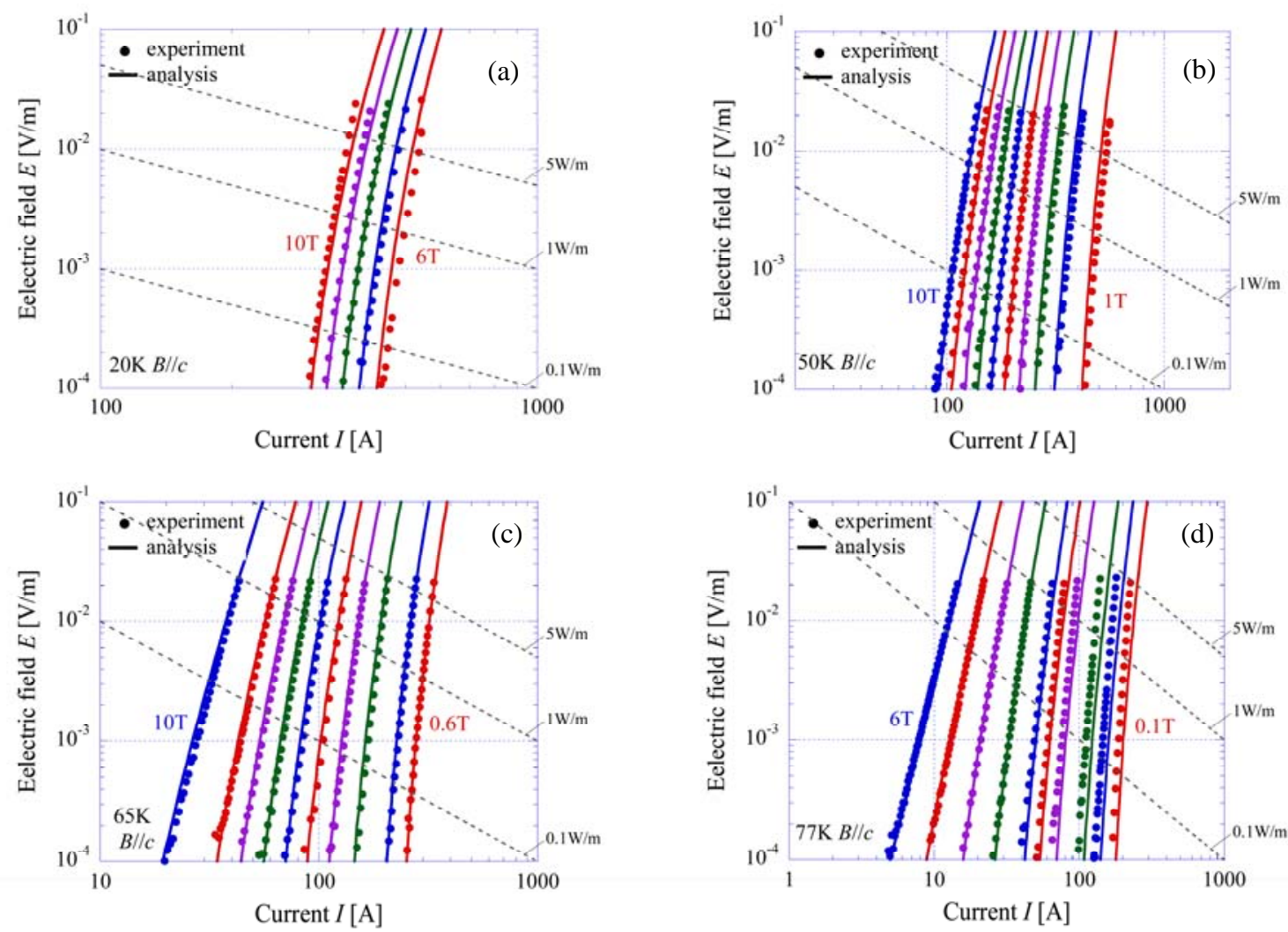

Fig. 3 E-I characteristics in a 4-mm-wide Gd-123 coated conductor. The measurement was done using a microbridge, then the current was calibrated for the 4-mm-wide full width tape. Solid curves are analytical expression whereas dots are measurements.

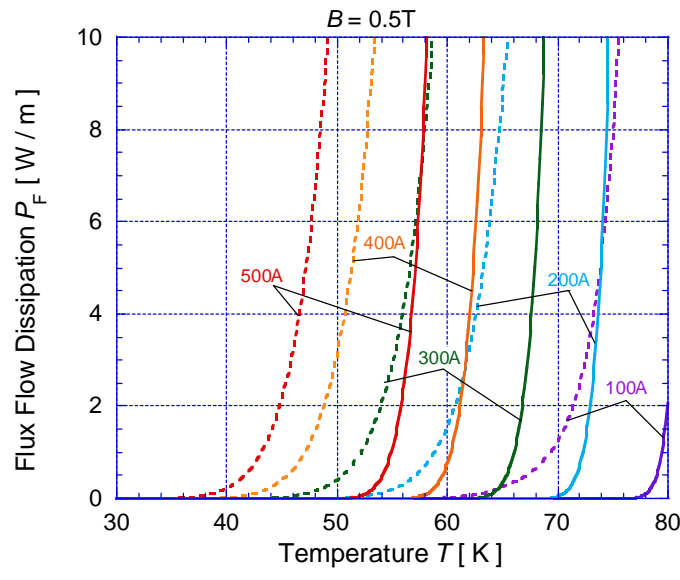

(a)

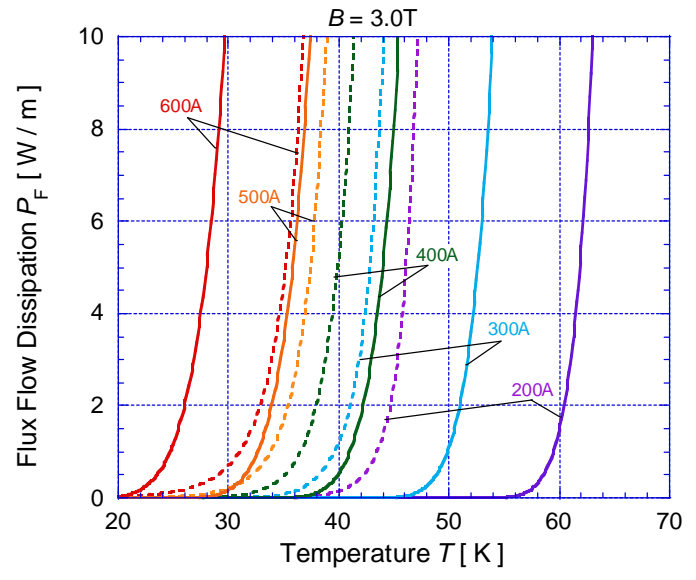

(b)

Fig. 4 Temperature dependent flux flow dissipation, $P_{\mathrm{F}}$, in the $3.8 \mathrm{~mm}$-wide Bi-2223 tape (broken lines) and 4 mm-wide Gd-123 CC (solid lines). (a) at around 65K, (b) at around 30K. 

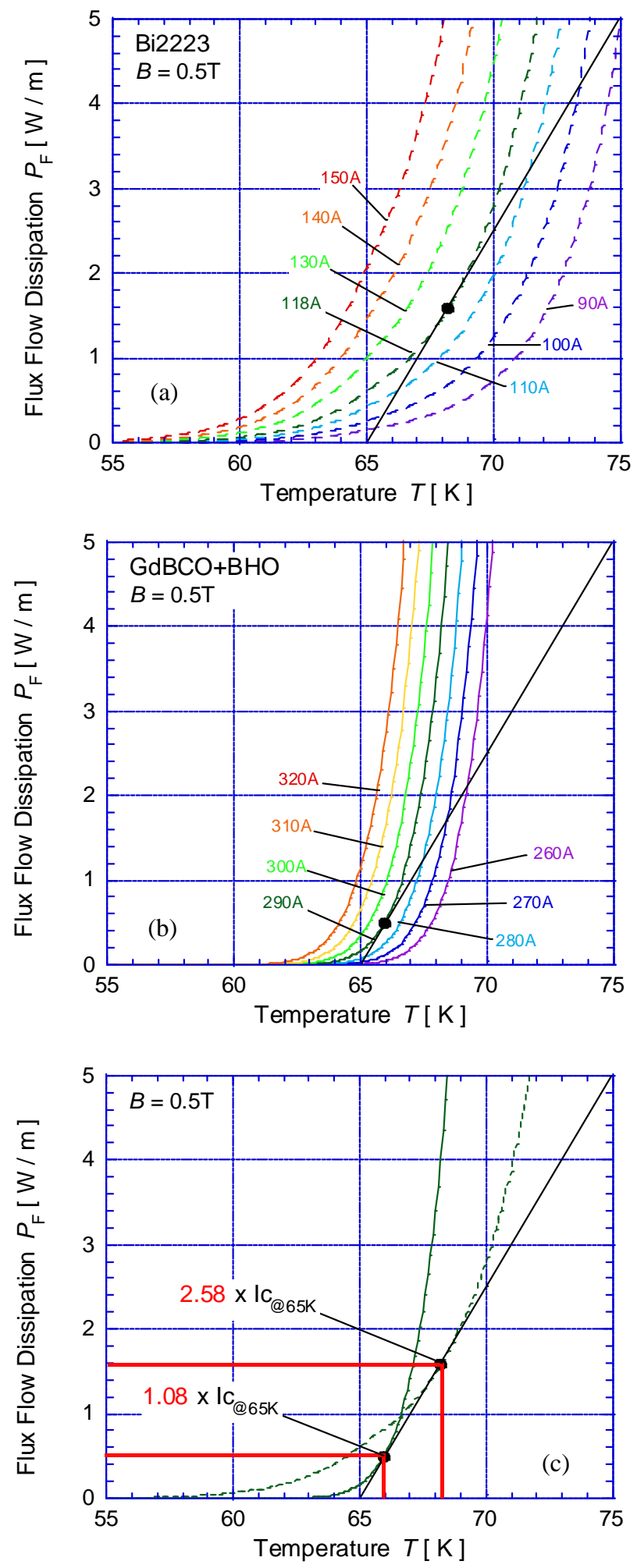

Fig. 5 Temperature dependence of bias current dependent flux flow dissipation under $0.5 \mathrm{~T}$ of external magnetic field perpendicular to the tape surface at $65 \mathrm{~K}$ of operation temperature. (a) Bi-2223 tape, (b) Gd-123 CC, (c) critical point for thermal runaway. 

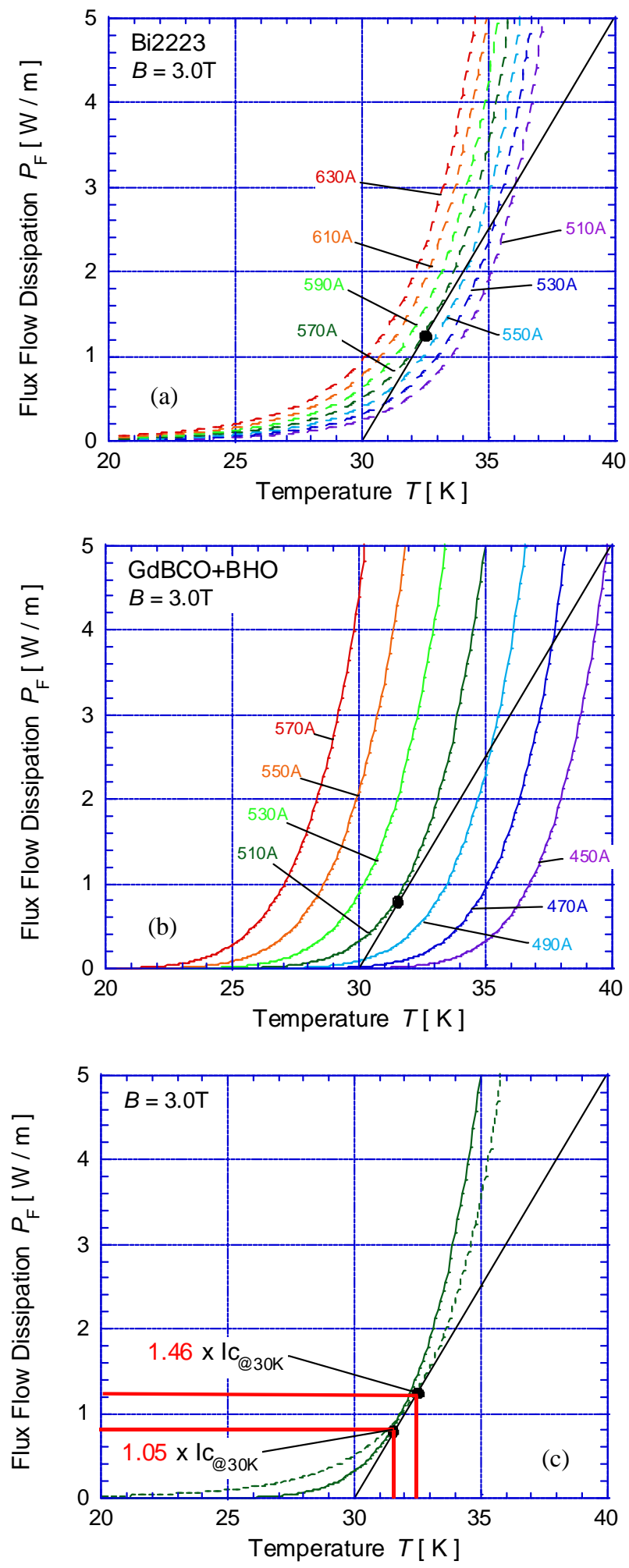

Fig. 6 Temperature dependence of bias current dependent flux flow dissipation under $3.0 \mathrm{~T}$ of external magnetic field perpendicular to the tape surface at $30 \mathrm{~K}$ of operation temperature. (a) Bi-2223 tape, (b) BHO doped Gd-123 CC, (c) critical point for thermal runaway. 


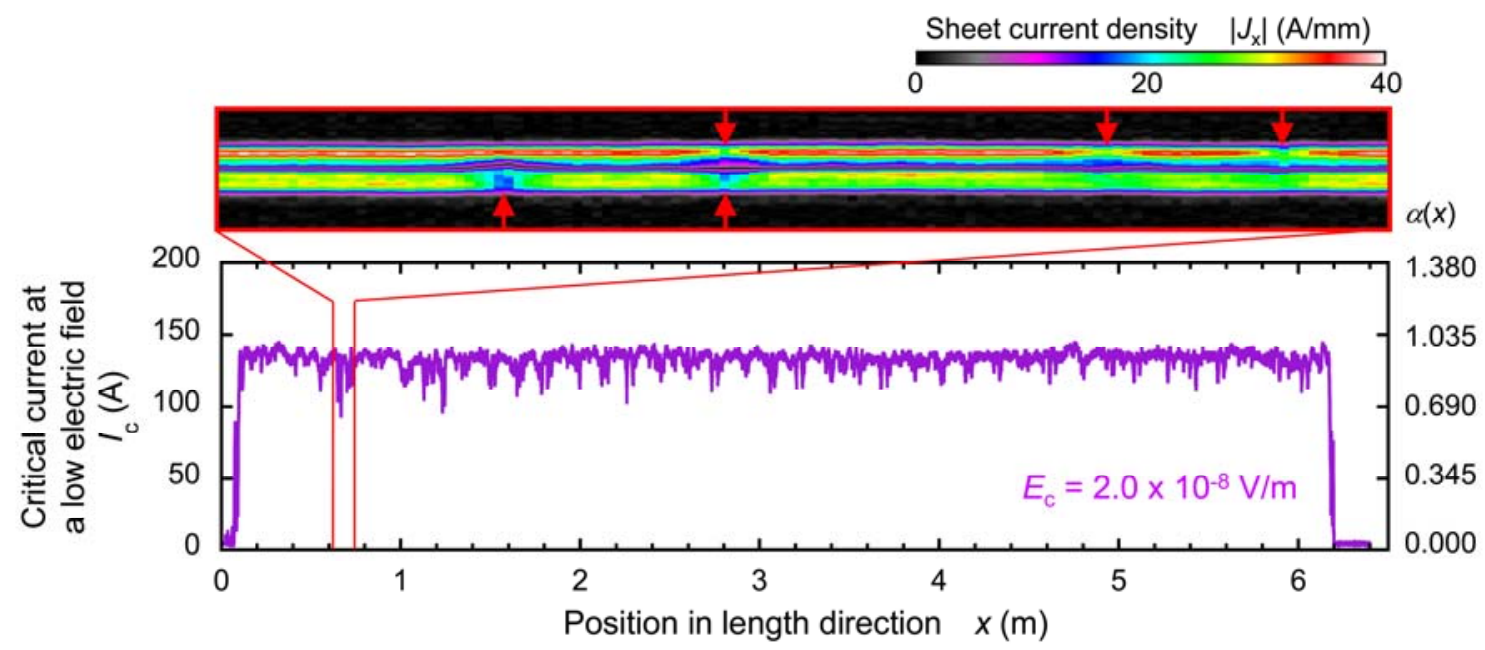

(a)

$q(\mathrm{~mW} / \mathrm{cm})$

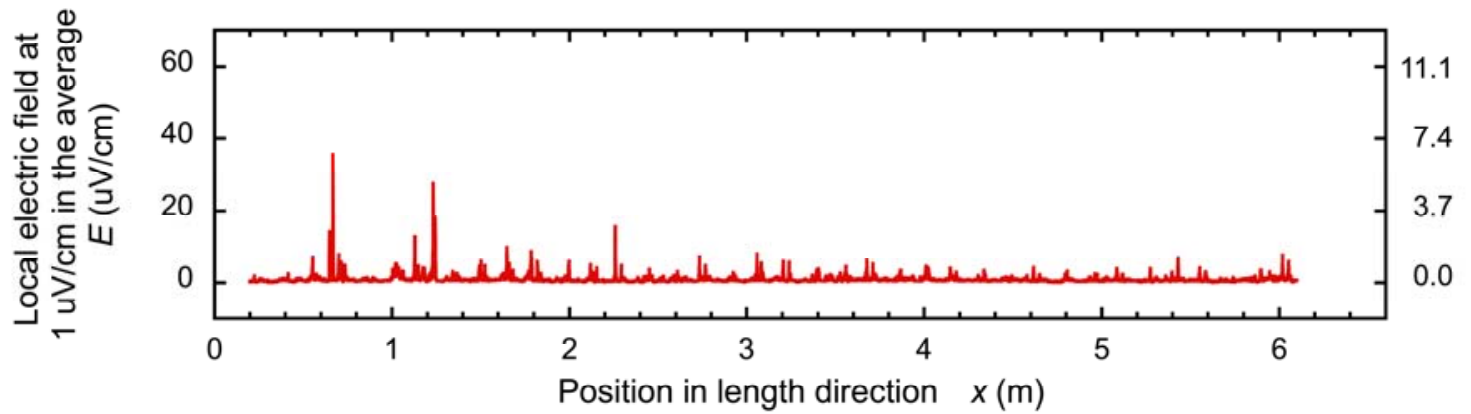

(b)

Fig. 7 (a) Longitudinal variation of local $I_{\mathrm{c}}$ in a 6-m-long Bi-2223 tape. The sample is the same lot studied in-field flux flow dissipation discussed in the preceding section. $\alpha$ in the right axis shows the change of effective cross-section area. The inset in the upper panel is enlarged image of longitudinal component of in-plane magnetization current distribution (sheet current density) visualized by the RTR-SHPM. (b) Spatial variation of local electric field when the global electric field reaches $1 \mu \mathrm{V} / \mathrm{cm}$. $q$ in the right axis indicates local dissipation. 


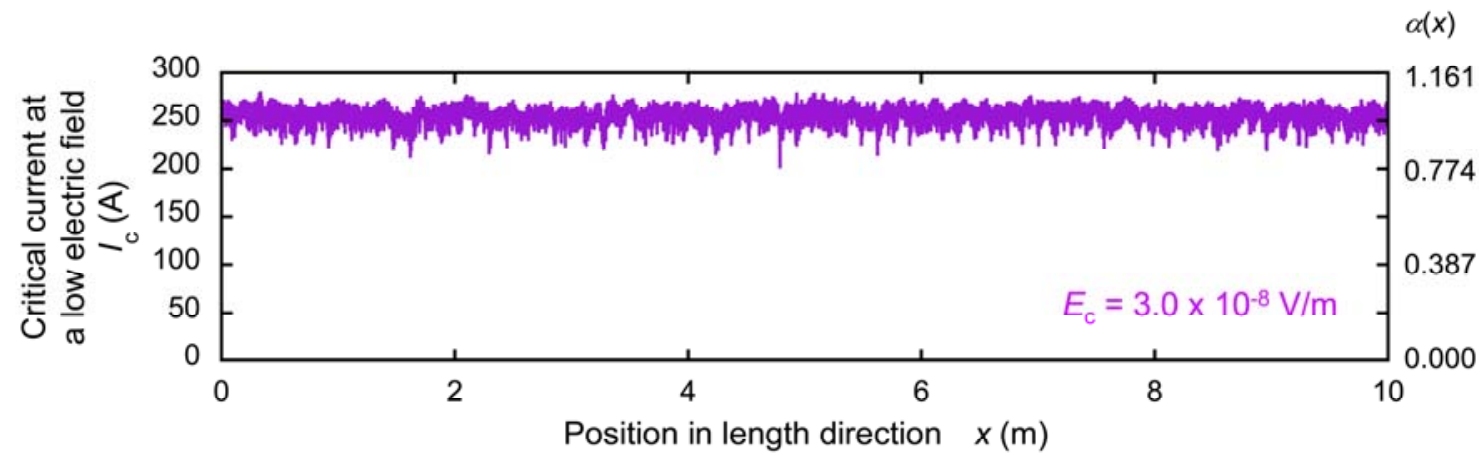

(a)

$q(\mathrm{~mW} / \mathrm{cm})$

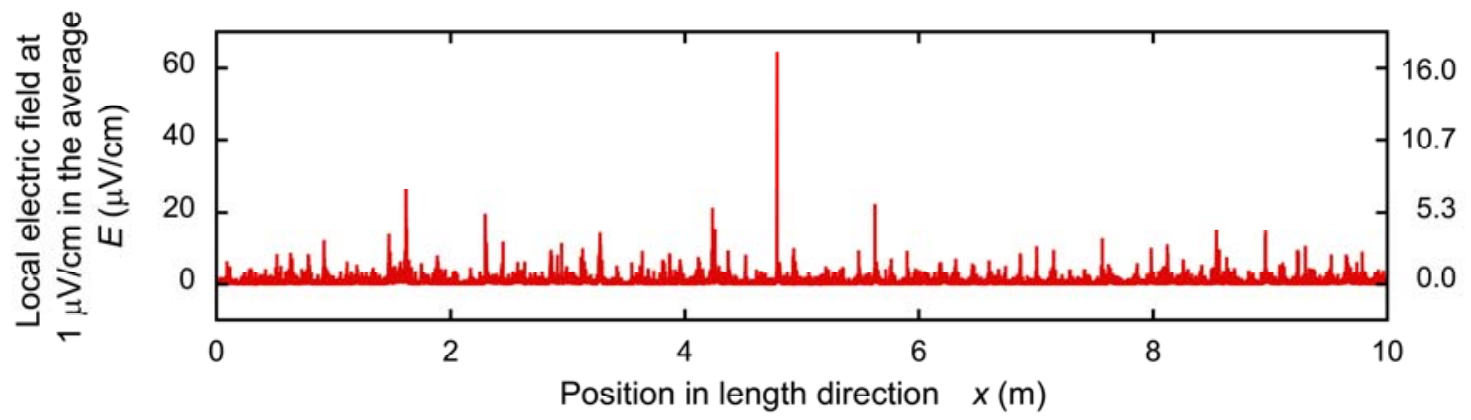

(b)

Fig. 8 (a) Longitudinal variation of local $I_{\mathrm{c}}$ in a 10-m-long Gd-123 CC tape. $\alpha$ in the right axis shows the change of effective cross-section area. (b) Spatial variation of local electric field when the global electric field reaches $1 \mu \mathrm{V} / \mathrm{cm}$. $q$ in the right axis indicates local dissipation. 


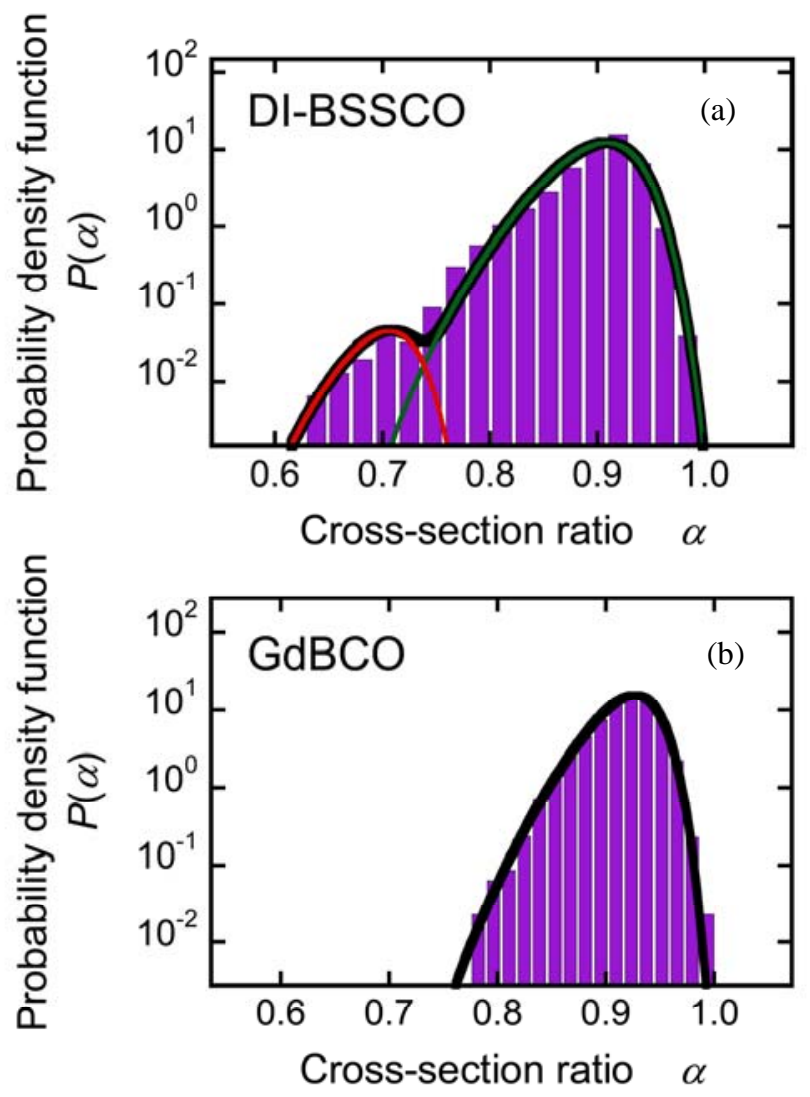

Fig. 9 Statistical distribution of effective superconducting area in the HTS tapes obtained from the RTR-SHPM. Solid curves are analytical expression by the Weibull function. Parameters of the functions were listed in Table 2. (a) Bi-2223 tape, (b) Gd-123 CC. 